\title{
PICEATANNOL AMELIORATES CISPLATIN-INDUCED HISTOLOGICAL AND BIOCHEMICAL ALTERATIONS IN RATS KIDNEY
}

\author{
SARA A. WAHDANa, SAMAR S. AZABa ${ }^{a}$, DOAA A. ELSHERBINYN", EBTEHAL EL-DEMERDASH ${ }^{a}$ \\ aPharmacology and Toxicology Department, Faculty of Pharmacy, Ain shams University, Cairo, Egypt \\ Email: ebtehal_dm@yahoo.com
}

Received: 04 Oct 2016 Revised and Accepted: 31 Aug 2017

\begin{abstract}
Objective: The present study was designed to investigate the effect of different doses of piceatannol (PIC) on cisplatin-induced biochemical and histological alterations in rat kidney.

Methods: Male Wistar rats received a single intraperitoneal (i. p.) injection of cisplatin (7 mg/kg). PIC was given in different daily doses (5, 10 and $20 \mathrm{mg} / \mathrm{kg}$ ) i. p., for seven days, starting two days before cisplatin injection. Nephrotoxicity was evaluated by means of measurement of blood urea nitrogen (BUN), serum creatinine and histopathological examination of the kidney. We also investigated the antioxidant effect of the most effective dose of PIC by measuring reduced glutathione (GSH) and lipid peroxides levels. Moreover, the ability of PIC to affect nuclear factor kappa B (NF-kB) expression was determined by immunohistochemical analysis.
\end{abstract}

Results: A single dose of cisplatin ( $7 \mathrm{mg} / \mathrm{kg}$ ) significantly increased BUN and creatinine levels, as well as relative kidney weight, compared to the control group. In addition, significant histopathological alterations including tubular necrosis, hemorrhage and casts formation were observed. PIC was given in different doses $(5,10$ and $20 \mathrm{mg} / \mathrm{kg}$ ) for $7 \mathrm{~d}$, starting $2 \mathrm{~d}$ before cisplatin injection. PIC dose $10 \mathrm{mg} / \mathrm{kg}$ was the most effective in preventing these alterations. PIC significantly increased GSH level and decreased lipids peroxidation compared to cisplatin group. Moreover, PIC significantly mitigated cisplatin-induced expression of NF-kB.

Conclusion: PIC has the potential to ameliorate cisplatin-induced renal injury.

Keywords: Cisplatin, Piceatannol, Nephrotoxicity, Antioxidants, NF-kB

(C) 2017 The Authors. Published by Innovare Academic Sciences Pvt Ltd. This is an open access article under the CC BY license (http://creativecommons.org/licenses/by/4. 0/) DOI: http://dx.doi.org/10.22159/ijpps.2017v9i10.15518

\section{INTRODUCTION}

Cisplatin is an effective and widely used chemotherapeutic agent [1] Nephrotoxicity represents one of its major and clinically significant side effects, limiting its clinical use [2]. The high prevalence of cisplatin-induced nephrotoxicity is well documented, occurring in about one-third of patients receiving cisplatin treatment [3]. Cisplatin-induced renal toxicity is dose-dependent, limiting the possibility of increasing dosages, which affects treatment effectiveness [4]. It has a direct toxic effect on renal tubules, vasculature and glomeruli. Multiple cellular mechanisms were found to be involved in cisplatin-induced renal damage, including oxidative stress, inflammatory response and activation of apoptotic pathways [5]. Clinically, renal toxicity begins with $20 \%$ to $40 \%$ decline in glomerular filtration, followed by increased serum levels of creatinine and blood urea nitrogen (BUN), hypomagnesemia and hypokalemia [1].

There is no specific treatment for the cisplatin-induced renal injury. The most important supportive measures may include adequate hydration, use of diuretics and electrolytes replacement [6]. Therefore, considerable efforts have been focused on exploring new compounds serving as protective treatments against cisplatininduced nephrotoxicity $[7,8]$.

Piceatannol (3,4',3',5-transtrihydroxystilbene) (PIC) is a natural analogue and a metabolite of resveratrol[9]. It was first isolated from the seeds of Euphorbia lagascae [10]. The most important sources of PIC in the diet are grapes, passion fruit, white tea, and Japanese knotweed [11]. PIC is an important naturally occurring polyphenolic stilbene, produced by plants in response to fungal infection, mechanical damage and ultra-violet irradiation. It can be consumed on a daily basis with no harmful effects on human body [12]. PIC is known as a potent scavenger of free radicals due to hydroxyl groups in its stilbene rings [13]. The anti-proliferative, anti-inflammatory and antioxidant properties of PIC were proved in multiple in vivo and in vitro studies [14-17]. One study showed that PIC is a potent enhancer of cisplatin sensitivity in ovarian cancer, potentially, through the modulation of several major determinants of chemoresistance [18]. However, its role as a protective agent against cisplatin-induced nephrotoxicity has not been investigated. Therefore, the present study was performed to investigate the potential protective effect of different doses of PIC against cisplatininduced nephrotoxicity in rats.

\section{MATERIALS AND METHODS}

\section{Animals}

Male Wistar rats weighing 200-250 g were purchased from the National Institute of Research, (Cairo, Egypt). Rats were kept in an air-conditioned atmosphere, at a temperature of $25{ }^{\circ} \mathrm{C}$ with alternatively $12 \mathrm{~h}$ light and dark cycles and kept on a standard diet ad libitum and water. Standard diet pellets (El-Nasr, Abu Zaabal, Egypt) containing not less than $20 \%$ protein, $5 \%$ fiber, $3.5 \%$ fat, $6.5 \%$ ash and a vitamin mixture. Few days before the experiment, animals were acclimatized to laboratory conditions. The study was approved by the ethical committee of Faculty of Pharmacy, Ain Shams University (Cairo, Egypt) (REC-ASU PhD No. 5).

\section{Drugs and chemicals}

Cisplatin was obtained from Mylan (Saint-Priest, France), dissolved in saline at a concentration $1 \mathrm{mg} / \mathrm{ml}$ and injected i. p. as a single dose of $7 \mathrm{mg} / \mathrm{kg}$. PIC (98\% pure) was purchased from Alexis Biochemicals (San Diego, CA, USA) and dissolved in dimethyl sulfoxide (DMSO) and normal saline $(0.9 \%)$ in a ratio $(2: 3)$. The protective effect of different doses of PIC $(5,10$ and $20 \mathrm{mg} / \mathrm{kg})$ against cisplatin-induced nephrotoxicity was screened by the assessment of nephrotoxicity markers. Serum creatinine and BUN were estimated using available commercial kits purchased from Endomedics Co. (Cairo, Egypt). GSH and lipid peroxides levels were determined using commercial kits obtained from Biodiagnostics Co. (Giza, Egypt). Rabbit polyclonal 
anti NF- $\kappa$ B p65 antibody was purchased from Thermo Fisher Scientific (USA) (Cat No. RB-9034-P). Bovine serum albumin, diaminobenzidine, tris base and DMSO were purchased from Sigma Chemical Co. (St. Louis, MO, USA). Ethanol, methanol, formaldehyde, dipotassium hydrogen phosphate $\left(\mathrm{K}_{2} \mathrm{HPO}_{4}\right)$ and potassium dihydrogen phosphate $\left(\mathrm{KH}_{2} \mathrm{PO}_{4}\right)$ were purchased from El Nasr Chemical Co. (Cairo, Egypt). All other chemicals were of the highest purity grade commercially available.

\section{Experimental design}

Screening the effective protective dose of PIC against cisplatininduced nephrotoxicity

A dose response screening study was conducted, in which animals were randomly divided into six groups (ten animals per group) and treated as follows; The first group was considered as control group and received the vehicle (DMSO and isotonic saline) (2:3), 0.2 $\mathrm{ml} / 200$ gm body weight (i. p.), once daily for 7 consecutive days and a single injection of isotonic saline $(0.2 \mathrm{ml} / 200$ gm body weight, i. p.) on the third day of the experiment. The second group was given the vehicle i. p., once daily for 7 consecutive days and a single injection of cisplatin $(7 \mathrm{mg} / \mathrm{kg}$, i. p.) on the third day of the experiment to induce nephrotoxicity. The third, fourth and the fifth groups received PIC at a dose of 5, 10 and $20 \mathrm{mg} / \mathrm{kg}$, respectively, i. p., once daily for 7 consecutive days and a single dose of cisplatin $7 \mathrm{mg} / \mathrm{kg}$ i. p. on the third day of the experiment. PIC was given $2 \mathrm{~h}$ prior to cisplatin injection [16]. The sixth group received PIC at a dose 20 $\mathrm{mg} / \mathrm{kg}$ i. p., once daily for 7 consecutive days.

At the end of the experiment, the number of dead animals and the change in animals' weight were recorded. Then, rats were anesthetized and blood samples were collected from the retroorbital plexus and allowed to clot. Serum was separated by centrifugation at $3000 \mathrm{rpm}$ for $10 \mathrm{~min}$ and was stored at- $80{ }^{\circ} \mathrm{C}$ until assayed for the biochemical parameters. Then rats were sacrificed and kidney tissues were dissected out and washed with ice-cold saline. Additionally, specimens from kidneys of each group were fixed in $10 \%$ formalin for histopathological examination.

Studying the possible mechanisms underlying the protective effect of PIC against cisplatin-induced nephrotoxicity

In order to determine the potential mechanism underlying the nephroprotective effect of PIC, another study was conducted using its most effective dose in which animals were divided randomly into 4 groups (ten animals per group) and treated as follows; The first group was considered as control group and given the vehicle (DMSO and isotonic saline) (2:3) i. p., once daily for 7 consecutive days and a single injection of isotonic saline i. p. on the third day of the experiment. The second group was given the vehicle i. p., once daily for 7 consecutive days and a single injection of cisplatin $(7 \mathrm{mg} / \mathrm{kg}$, i. p.) on the third day of the experiment to induce nephrotoxicity. The third, group received PIC at a dose $10 \mathrm{mg} / \mathrm{kg}$, i. p., once daily for 7 consecutive days and a single dose of cisplatin $7 \mathrm{mg} / \mathrm{kg}$ i. p. on the third day of the experiment. The fourth group received PIC at a dose $10 \mathrm{mg} / \mathrm{kg}$ i. p., once daily for 7 consecutive days.

At the end of the experiment, kidney tissues were dissected and washed with ice-cold saline. Portions of the kidney tissues were homogenized in $0.1 \mathrm{M}$ phosphate buffer $(\mathrm{pH}$ 7.4) producing $10 \%$ homogenate. The homogenates were centrifuged at $4000 \mathrm{rpm}$ at $4{ }^{\circ} \mathrm{C}$ for $15 \mathrm{~min}$ then aliquots of supernatants were separated and used for biochemical analyses. Additionally, specimens from kidneys of each group were fixed in $10 \%$ formalin for immunohistochemical analysis.

\section{Assessment of nephrotoxicity markers}

Serum creatinine and BUN were estimated using available commercial kits (Endomedics Co. Cairo, Egypt). Briefly, creatinine was determined according to Jaffe-kinetic method which depends on the measurement of the rate of formation of a colored complex between creatinine and alkaline picrate [19]. BUN determination depends on the formation of a green colored compound due to the reaction between urea and $\mathrm{Na}$ nitroprusside in the presence of urease enzyme [20]. The intensity of this color is proportional to BUN. Spectrophotometric analyses were performed using UV-visible spectrophotometer (UV-1601) (Shimadzu Co, Japan). Relative kidney weight was calculated according to the formula: (kidney weight/total body weight) $\times 100$.

\section{Histopathological examination}

For light microscopy, kidney specimens were fixed in $10 \%$ formalin for $24 \mathrm{~h}$ and then washed with tap water. Serial dilutions of alcohol (methyl, ethyl and absolute ethyl alcohol) were used for dehydration. Specimens were cleared in xylene embedded in paraffin at $56^{\circ} \mathrm{C}$ in hot air oven for $24 \mathrm{~h}$. Paraffin tissue blocks were prepared for sectioning at 4 microns thickness by sledge microtome. The obtained tissue sections were collected on glass slides and deparaffinized. Finally, sections were stained with hematoxylin and eosin ( $\mathrm{H}$ and $\mathrm{E}$ ) and examined under the light microscope (Olympus BX-50 Olympus Corporation, Tokyo, Japan). [21].

\section{Assessment of oxidative stress markers}

Assessments of reduced glutathione (GSH) and lipid peroxides levels were performed using commercial kits (Biodiagnostics Co. Giza, Egypt). Briefly, GSH assessment was based on the reduction of 2nitrobenzoic acid by GSH to produce a yellow compound. The absorbance of the yellow colored product was determined at 405 $\mathrm{nm}$. Lipid peroxidation was estimated spectrophotometrically by measuring malondialdehyde (MDA) level. Thiobarbituric acid (TBA) reacts with MDA in acidic medium at temperature $95^{\circ} \mathrm{C}$ for $30 \mathrm{~min}$ to form a thiobarbituric acid reactive product. The absorbance of the pink colored product was determined at $535 \mathrm{~nm}$. Spectrophotometric analyses were performed using UV-visible spectrophotometer (UV-1601) (Shimadzu Co, Japan).

\section{Immunohistochemical detection of NFkB (p65)}

Paraffin-embedded tissue sections, of $3 \mu \mathrm{m}$ thickness, were rehydrated first in xylene and then in graded ethanol solutions. The slides were then blocked with $5 \%$ bovine serum albumin in trisbuffered saline for $2 \mathrm{~h}$. The sections were then immunostained with the rabbit polyclonal anti NF- $\mathrm{KB}$ p65 primary antibody (Thermo Fisher Scientific, Cat No. RB-9034-P), at a concentration of $1 \mu \mathrm{g} / \mathrm{ml}$ containing $5 \%$ bovine serum albumin in tris-buffered saline and incubated overnight at $41{ }^{\circ} \mathrm{C}$. After washing the slides with trisbuffered saline, the sections were incubated with goat anti-rabbit secondary antibody. Sections were then washed with tris buffered saline and incubated for $5-10 \mathrm{~min}$ in a solution of $0.02 \%$ diaminobenzidine containing $0.01 \% \mathrm{H}_{2} \mathrm{O}_{2}$. Counter staining was performed using hematoxylin stain. Slides were examined with the light microscope (Olympus BX-50 Olympus Corporation, Tokyo, Japan). Immunohistochemical quantification was carried out using image analysis software (Image J, 1.46a, NIH, USA).

\section{Statistical analysis}

Data were presented as mean \pm SD Statistical analysis was performed using one-way analysis of variance (ANOVA), followed by TukeyKramer as a post Hoc test. Two-tailed Student's t-test was used for statistical comparisons between two groups. The 0.05 level of probability was used as the criterion for significance. All statistical analyses were performed using Instat software package (version 3). Graphs were sketched using GraphPad Prism software (version 5).

\section{RESULTS}

\section{Mortality, body weight and relative kidney weight}

The results of nephrotoxicity markers including mortality, total body weight and relative kidney weight were shown in table 1 . In cisplatin group, three animals died and a significant decrease in the body weight of rats by $17 \%$, as compared to their initial weight associated with a significant increase in relative kidney weight by $32 \%$, as compared to the control group, were observed. Two animals died in the group treated concomitantly with PIC $(5 \mathrm{mg} / \mathrm{kg})$. No animal death was observed in rats co-treated with 10 and $20 \mathrm{mg} / \mathrm{kg}$ PIC. Other treated groups did not show any significant change in their body weight, as compared to their initial weight or in relative kidney weight, as compared to the control group. Concomitant treatment with PIC $(5,10$ and $20 \mathrm{mg} / \mathrm{kg})$ caused a significant reduction in relative kidney weight by $22 \%, 30 \%$ and $30 \%$, respectively, as compared to cisplatin-treated group. 
Table 1: Effects of different doses of piceatannol (PIC) on body weight and relative kidney weight in cisplatin-treated rats

\begin{tabular}{lllll}
\hline Groups & No. of dead rats & \multicolumn{2}{l}{ Body weight (gms) } & Relative kidney weight (\%) \\
\cline { 2 - 4 } & & Before treatment & After treatment & $0.68 \pm 0.07$ \\
\hline Control & $0 / 10$ & $221 \pm 40.14$ & $242 \pm 38.3$ & $1 \pm 0.178^{\mathrm{a}}$ \\
Cisplatin & $3 / 10$ & $214.9 \pm 45.8$ & $178.9 \pm 16.3^{*}$ & $0.78 \pm 0.16^{\mathrm{b}}$ \\
Cisplatin+PIC (5 mg/kg) & $2 / 10$ & $240.7 \pm 45.9$ & $202.6 \pm 25.7$ & $0.7 \pm 0.05^{\mathrm{b}}$ \\
Cisplatin+PIC (10 mg/kg) & $0 / 10$ & $260 \pm 57.9$ & $215 \pm 32.8$ & $0.7 \pm 0.04^{\mathrm{b}}$ \\
Cisplatin+PIC (20 mg/kg) & $0 / 10$ & $230 \pm 34.6$ & $210.8 \pm 43.5$ & $0.64 \pm 0.02^{\mathrm{b}}$ \\
PIC (20 mg/kg) & $0 / 10$ & $210.5 \pm 52.7$ & $220 \pm 39.7$ & \\
\hline
\end{tabular}

Data were presented as means $\pm S D(n=10)$, a: significantly different from the control group; $b$ : significantly different from cisplatin group. $\mathrm{P}<0.05$ using ANOVA followed by Tukey-Kramer as a post-hoc test. *Significantly different from pre-treated group, $\mathrm{P}<0.05$ using student paired t-test, PIC: Piceatannol.

\section{Serum creatinine and BUN}

Cisplatin treatment caused a significant increase in serum creatinine and BUN by $564 \%$ and $764 \%$, respectively, compared to the control group. Concomitant treatment with PIC $(5,10$ and $20 \mathrm{mg} / \mathrm{kg})$ significantly ameliorated nephrotoxicity markers, indicated by a significant reduction in serum creatinine levels by 27,55 and $53 \%$, respectively, as compared to cisplatin treated group. Similarly, treatment with PIC $(5,10$ and $20 \mathrm{mg} / \mathrm{kg})$ significantly reduced BUN levels by 33,58 and $50 \%$, respectively, compared to cisplatin group. In addition, serum creatinine and BUN in animals treated with PIC (10 and $20 \mathrm{mg} / \mathrm{kg}$ ) were significantly lower than those in the group treated with PIC $(5 \mathrm{mg} / \mathrm{kg}$ ). On the other hand, there was no significant difference between the two groups treated with PIC (10 $\mathrm{mg} / \mathrm{kg})$ and PIC $(20 \mathrm{mg} / \mathrm{kg})$ in all nephrotoxicity markers. Treatment of rats with PIC alone did not show any significant change in all nephrotoxicity parameters, as compared to the control group (fig. 1).
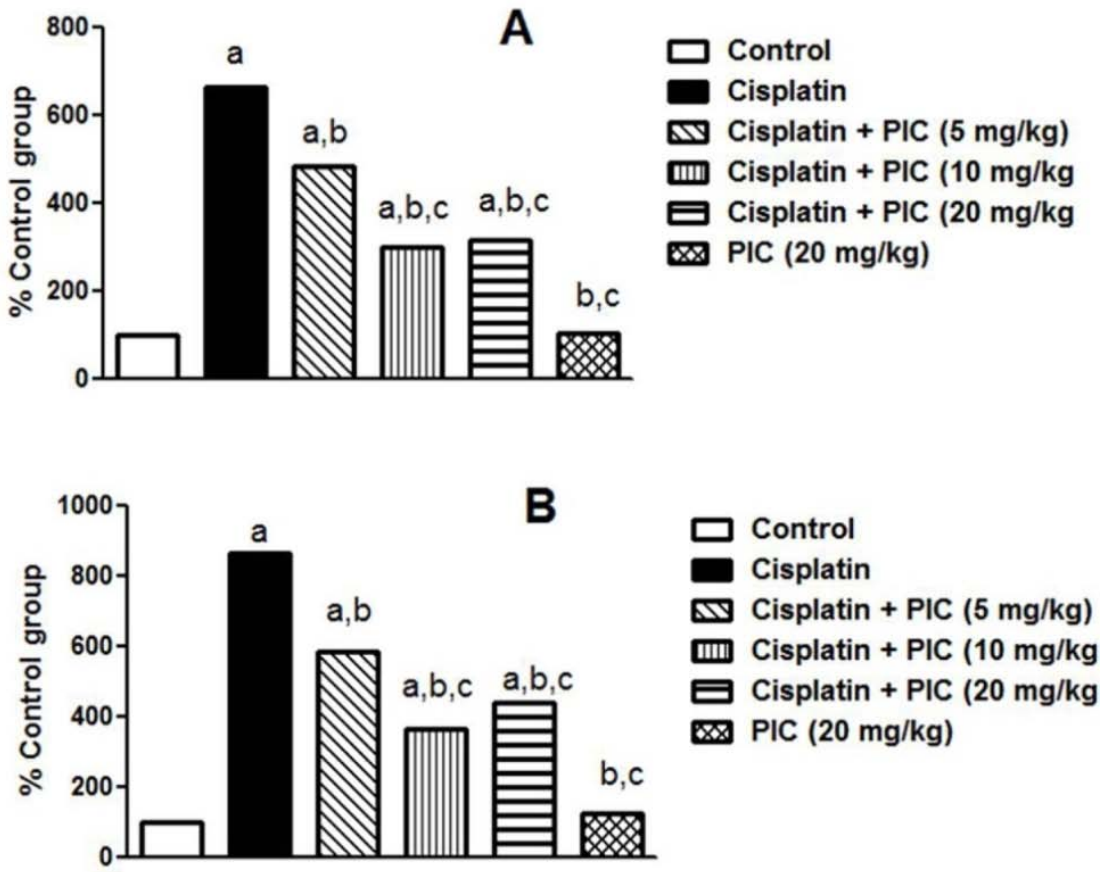

Fig. 1: Effects of different doses of piceatannol (PIC) on (A) serum creatinine and (B) BUN in cisplatin-treated rats, Data were presented as $\%$ of control group, a: significantly different from the control group; b: significantly different from cisplatin group; c: significantly different from cisplatin and PIC $(5 \mathrm{mg} / \mathrm{kg})$ treated group. $\mathrm{P}<0.05$ using ANOVA followed by Tukey-Kramer as a post-hoc test

\section{Histopathological examination}

Histopathological alterations in kidney specimens from different treatment groups were shown in fig. 2. Kidney specimens from the control rats (fig. $2 \mathrm{~A})$ and rats treated with PIC $(20 \mathrm{mg} / \mathrm{kg})$ alone (fig. 2B) showed a normal histological structure of the glomeruli and renal tubules. Treatment of rats with cisplatin caused severe congestion in the cortical blood vessels, as well as in the glomerular tufts and was associated with periglomerular inflammatory cells aggregation (fig. 2C). Degeneration in the tubular epithelial cells and eosinophilic casts formation in the tubular lumen were also observed in the cortical portion (fig. 2D). Kidney specimens from rats treated with PIC $(5 \mathrm{mg} / \mathrm{kg})$ concomitantly with cisplatin showed mild reduction in cisplatin-induced pathological alterations. Focal inflammatory cells, tubular necrosis, homogenous eosinophilic casts formation and cystic dilatation were observed (fig. 2E). Concomitant treatment of rats with PIC at higher doses $(10$ and $20 \mathrm{mg} / \mathrm{kg})$ ameliorated the pathological changes induced by cisplatin and restored the normal histological structure of the glomeruli and tubules (fig. $2 \mathrm{~F}$ and G). Only mild focal hemorrhage in the cortex was detected in the kidney specimens from the group pre-and co-treated with PIC $(10 \mathrm{mg} / \mathrm{kg})$ (fig. $2 \mathrm{~F})$. Various aspects of histopathological alterations in kidney specimens which were graded, as shown in table 2 . 


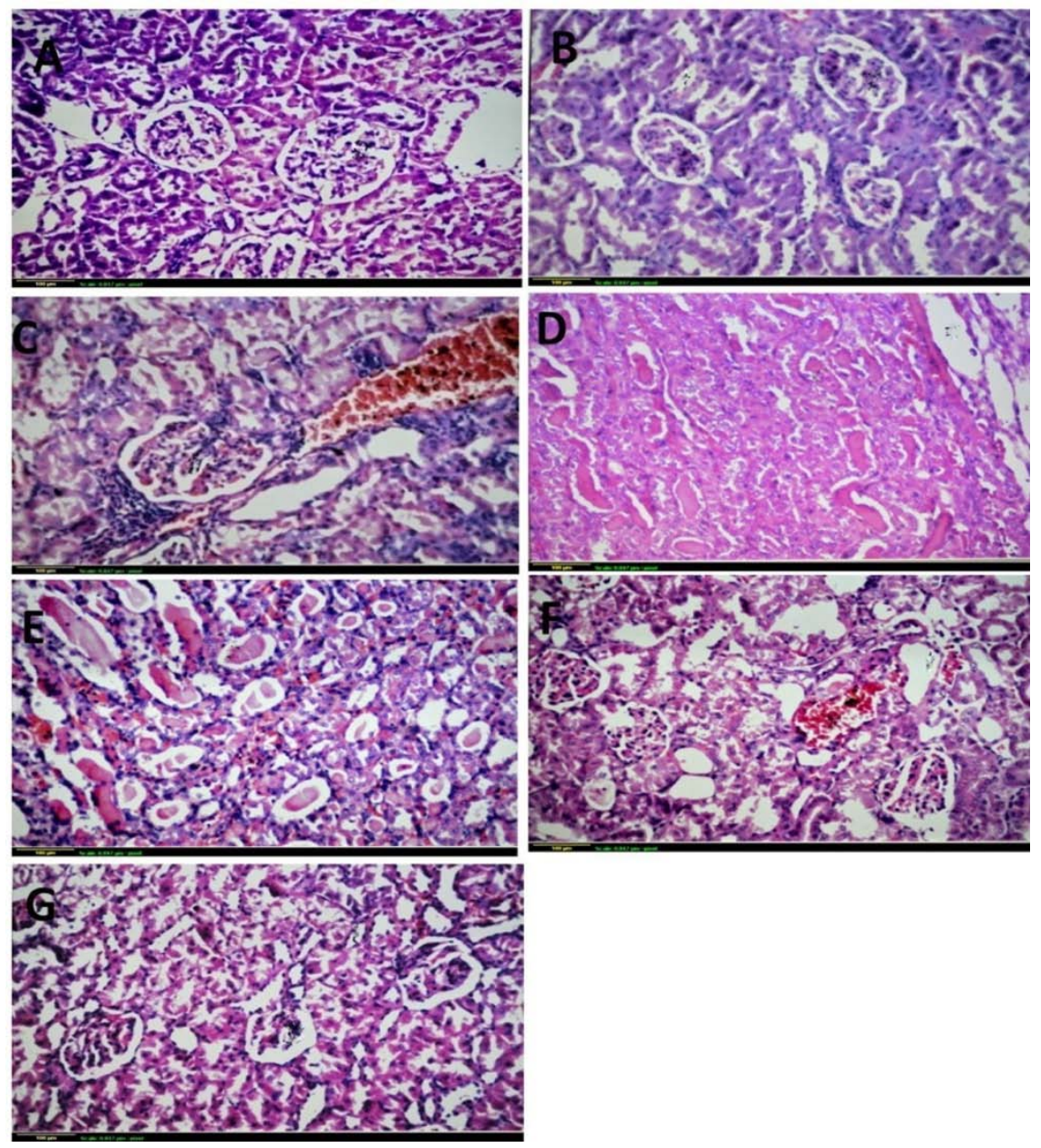

Fig. 2: Effects of different doses of piceatannol (PIC) on cisplatin-induced histological alterations of the kidney tissue, Representative photomicrographs of kidney sections stained with hematoxylin-eosin stain (magnification $\times 40)(A)$ control group, (B) PIC alone (20 $\mathrm{mg} / \mathrm{kg}$ ) treated group, (C and D) Cisplatin treated group, (E) Cisplatin and PIC (5 mg/kg) treated group, (F) Cisplatin and PIC (10 mg/kg) treated group and (G) Cisplatin and PIC $(20 \mathrm{mg} / \mathrm{kg})$ treated group

(A) and (B) showed a normal structure of glomeruli and tubules. (C) and (D) showed cisplatin-induced degeneration in tubular epithelial lining, hemorrhage, luminal dilatation and eosinophilic casts formation. (E) Cisplatin and PIC (5 mg/kg) co-treated group showed mild reduction in cisplatin-induced pathological alterations. Tubular necrosis and eosinophilic casts formation were observed. (F) and (G) showed that concomitant treatment of rats with PIC at higher doses ( 10 and $20 \mathrm{mg} / \mathrm{kg}$ ) ameliorated the pathological changes induced by cisplatin injection and restored the normal histological structure of the glomeruli and tubules. Mild focal hemorrhage in the cortex was detected in the kidney specimens from the group co-treated with PIC $(10 \mathrm{mg} / \mathrm{kg})(\mathrm{F})$.

Table 2: Aspects of histopathological alterations in kidney specimens

\begin{tabular}{llll}
\hline Group & Degeneration & Necrosis & Hemorrhage \\
\hline Control & - & - & - \\
Cisplatin & +++ & ++ & ++ \\
Cisplatin+PIC & + & + & - \\
$(5 \mathrm{mg} / \mathrm{kg})$ & - & - & + \\
Cisplatin+PIC & - & & - \\
$(10 \mathrm{mg} / \mathrm{kg})$ & - & - & - \\
Cisplatin+PIC & & - & - \\
$(20 \mathrm{mg} / \mathrm{kg})$ & - & - & - \\
PIC $(20 \mathrm{mg} / \mathrm{kg})$ & - & & - \\
\hline
\end{tabular}

$+++($ severe $),++($ moderate $),+($ mild $),-($ nil)., PIC: Piceatannol.

\section{Effect of PIC on oxidative stress markers}

Cisplatin significantly depleted GSH by $64 \%$ and increased MDA levels by $40 \%$, as compared to control group. In contrast, pre-and cotreatment of rats with the most effective dose of PIC $(10 \mathrm{mg} / \mathrm{kg})$ showed a significant increase in GSH level by $214 \%$ and a significant decrease in MDA level by $21 \%$, as compared to cisplatin group. Animals treated with PIC alone did not show any significant alterations in both GSH and MDA levels, as compared to the control group (fig. 3A and 3B). 
A

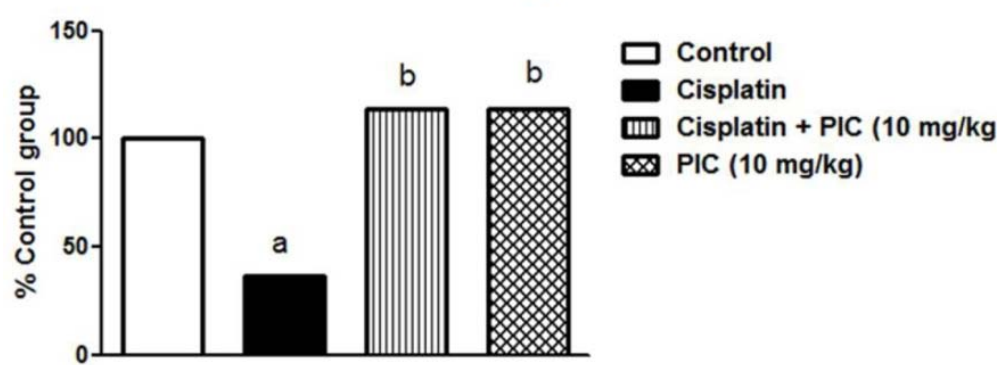

B
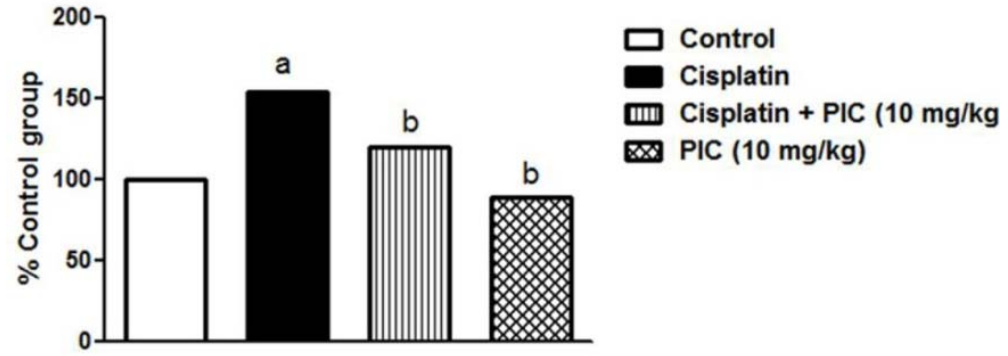

Fig. 3: Effect of piceatannol (PIC) on oxidative stress markers in cisplatin-treated rats. (A)Reduced glutathione level (GSH). (B) Lipid peroxidation expressed a malondialdehyde level (MDA), Data were presented as \% of control. a or b: significantly different from the control or cisplatin group respectively at $\mathrm{P}<0.05$ using ANOVA followed by Tukey-Kramer as a post-hoc test

\section{Effect of PIC on NF-kB (p65) expression}

The expression of the inflammatory marker NF-kB was estimated using immunohistochemical staining. The control group showed minimal immunostaining. No change in NF-kB expression was observed in the group treated with PIC alone. Cisplatin treatment increased the expression of NF-kB, as shown by the intense brown staining. Concomitant treatment with PIC $(10 \mathrm{mg} / \mathrm{kg})$ significantly mitigated cisplatin-induced expression of NF-kB. The immunohistochemical staining of NF-kB was quantified as the optical density of the stained regions using the image analysis software (fig. 4).
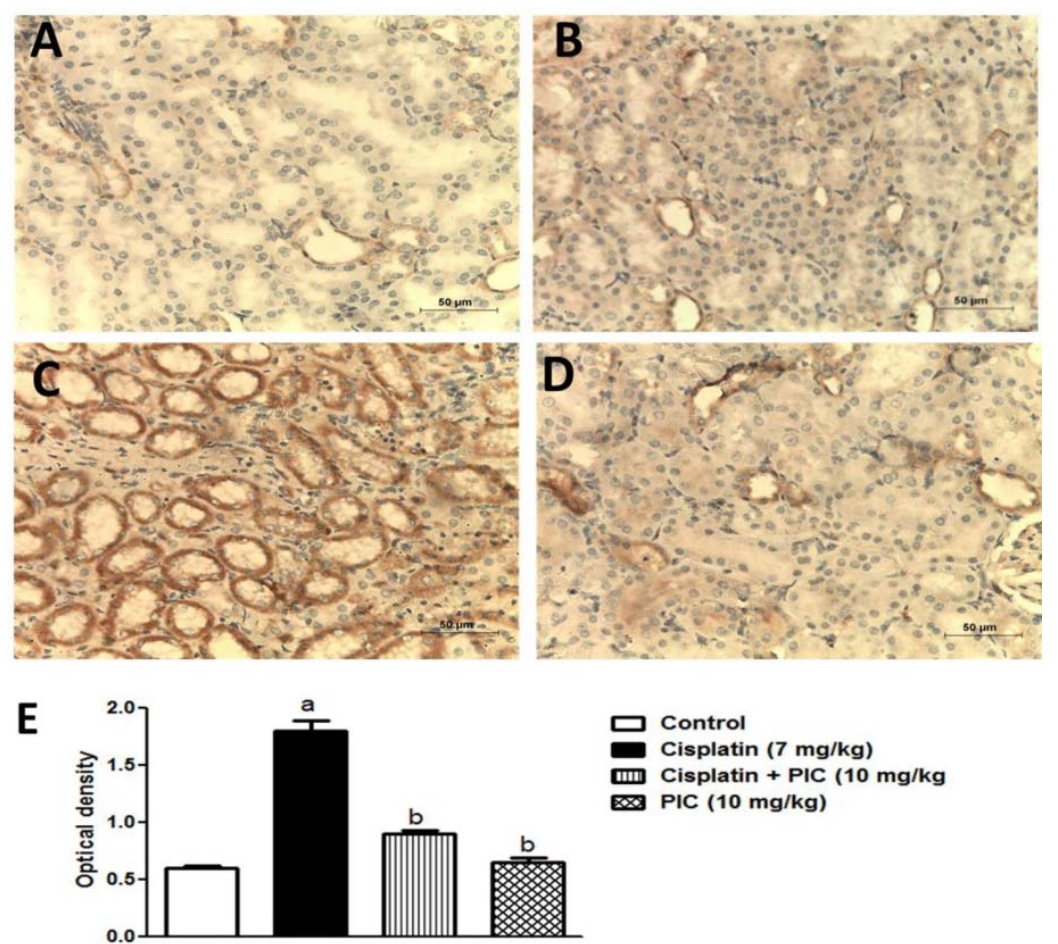

InI Cisplatin + PIC (10

PIC $(10 \mathrm{mg} / \mathrm{kg})$

Fig. 4: Effect of piceatannol (PIC) NF-KB (p65) expression in cisplatin-treated rats, Control (A) and PIC alone (B) groups: negative immunostaining, Cisplatin (C): extensive expression, Cisplatin+PIC (D): mild immunostaining. (E): Quantitative image analysis for immunohistochemical staining were expressed as optical densities across 10 different fields for each section. Data were presented as means \pm SD $(n=8)$. a or $b$ : significantly different from the control or cisplatin group respectively, at $P<0.05$ using ANOVA followed by Tukey-Kramer as a post-hoc test 


\section{DISCUSSION}

Cisplatin is a highly effective chemotherapeutic agent, used for the treatment of a wide spectrum of solid tumors but unfortunately, it causes dose-dependent nephrotoxicity that may lead to dose reduction or withdrawal. Nowadays, the usage of natural products as a protective strategy against cisplatin-induced nephrotoxicity represents a great scope of pharmacological research[22]. The current study provides an evidence of the protective effect of PIC against cisplatin-induced nephrotoxicity.

A dose-response study was conducted to choose the optimal dose of PIC for further investigations. Cisplatin was given i. p. as a single dose (7 $\mathrm{mg} / \mathrm{kg}$ ) causing significant alterations in nephrotoxicity markers; an increase in serum creatinine, BUN and relative kidney weight, as well as a decrease in total body weight. Increased mortality rate was also observed. The aforementioned abnormalities and biochemical changes were further confirmed by histological examination. It is well documented that cisplatin penetrates the tubular cells and reaches high concentrations in the proximal tubular cells of the inner renal cortex and outer medulla (S3 segment). Therefore, these areas are the major sites for cisplatin-induced renal injury, which in turn, leads to injury of other tubular areas including the distal and collecting tubules[23]. In the present study, cisplatin treatment caused severe nephrotoxic manifestations including degeneration of cortical epithelium, congestion, hemorrhage and necrosis, as well as eosinophilic casts formation in the lumen of proximal and distal convoluted tubules. Proximal convoluted tubules showed necrosis and degeneration of epithelial lining cells. These results were in agreement with previous studies [24-27].

Treatment of rats with $10 \mathrm{mg} / \mathrm{kg}$ PIC i. p. for $7 \mathrm{~d}$, starting two days before cisplatin single dose, significantly ameliorated the aforementioned cisplatin-induced alterations in kidney, as compared to the lowest dose $(5 \mathrm{mg} / \mathrm{kg})$. The highest dose $(20 \mathrm{mg} / \mathrm{kg})$ showed no significant benefit over the middle dose $(10 \mathrm{mg} / \mathrm{kg})$. In addition, histological examination confirmed the previous results. Based on these data, PIC in the dose $10 \mathrm{mg} / \mathrm{kg}$ has proven efficacy in protecting against cisplatin-induced nephrotoxicity and was used to assess the possible mechanisms underlying this nephroprotective effect.

Oxidative stress is highly involved in the pathogenesis of cisplatininduced kidney injury[28]. Cisplatin induces ROS production in renal epithelial cells, mainly by decreasing the activity of antioxidant enzymes and by GSH depletion which directly act on cellular components, including lipids, proteins and DNA which affect their structure and function [29]. In the present study, cisplatin injection $(7 \mathrm{mg} / \mathrm{kg})$ induced oxidative stress demonstrated by a significant depletion of reduced glutathione level and significant increase of lipid peroxidation in renal tissues, as compared to control group. These results were in agreement with previous studies [30-32]. In contrast, PIC restored reduced glutathione to normal levels and significantly decreased the extent of lipid peroxidation. The antioxidant effect of PIC was proven in previous studies [33, 34].

Inflammation plays a major role in the pathogenesis of cisplatin-induced nephrotoxicity, where a myriad of proinflammatory cytokines and chemokines are induced[1]. NF- $\mathrm{BB}$ can mediate inflammatory response, by affecting the expression of a large number of proinflammatory mediators such as TNF- $\alpha$, COX-2 and inducible nitric oxide[35]. Cisplatininduced ROS production activates NF- 0 B, which in turn induces the production of proinflammatory cytokines [36]. In the current study, the cisplatin-treated group showed a pro-inflammatory response as evidenced by significant increase in NF-kB expression. In contrast, concomitant treatment with PIC significantly decreased NF-kB expression and hence inhibited the downstream inflammatory cascade. In this regard, studies have shown that PIC could suppress NF- $\kappa B$ activation by different inflammatory inducers such as H2O2, lipopolysaccharides (LPS), okadaic acid, and ceramide [14]. In addition, our results were in accordance with previous studies that have reported the anti-inflammatory effect of PIC and its ability to diminish the production of inflammatory cytokines $[15,37]$.

\section{CONCLUSION}

These findings indicate for the first time that PIC may have a potential protective effect against cisplatin-induced renal injury, by restoring creatinine and BUN to normal levels and reversing the histopathological alterations in the kidney. A dose $10 \mathrm{mg} / \mathrm{kg}$ was the most effective and demonstrated an antioxidant effect against cisplatin-induced oxidative stress. Moreover, PIC significantly decreased NF- قB expression. Further studies are required to determine the possible underlying mechanisms of the nephroprotective effect of PIC, in terms of oxidative, inflammatory and apoptotic markers.

\section{CONFLICT OF INTERESTS}

The authors declared no potential conflicts of interest with respect to the research, authorship, and/or publication of this article.

\section{REFERENCES}

1. Miller RP, Tadagavadi RK, Ramesh G, Reeves WB. Mechanisms of cisplatin nephrotoxicity. Toxins (Basel) 2010;2:2490-518.

2. Santos NA, Catão CS, Martins NM, Curti C, Bianchi ML, Santos AC. Cisplatin-induced nephrotoxicity is associated with oxidative stress, redox state unbalance, impairment of energetic metabolism and apoptosis in rat kidney mitochondria. Arch Toxicol 2007;81:495-504.

3. Arany I, Safirstein RL. Cisplatin nephrotoxicity. Semin Nephrol 2003;23:460-4.

4. Peres LA, da Cunha AD Jr. Acute nephrotoxicity of cisplatin: molecular mechanism. J Bras Nefrol 2013;35:332-40.

5. Sánchez-González PD, López-Hernández FJ, López-Novoa JM, Morales AI. An integrative view of the pathophysiological events leading to cisplatin nephrotoxicity. Crit Rev Toxicol 2011;41:803-21.

6. Ozkok A, Edelstein CL. Pathophysiology of cisplatin-induced acute kidney injury. BioMed Res Int 2014;1:1-17.

7. Khan SA, Priyamvada S, Khan W, Khan S, Faroog N, Yusufi AN. Studies on the protective effect of green tea against cisplatin induced nephrotoxicity. Pharmacol Res 2009;60:382-91.

8. Sindhu G, Nishanthi E, Sharmila R. Nephroprotective effect of vanillic acid against cisplatin induced nephrotoxicity in wistar rats: a biochemical and molecular study. Env Toxicol Pharmacol 2015;39:392-404.

9. Piotrowska H, Kucinska M, Murias M. Biological activity of piceatannol: leaving the shadow of resveratrol. Mutat Res 2012;750:60-82.

10. Ferrigni NR, McLaughlin JL, Powell RG, Smith Jr CR. Use of potato disc and brine shrimp bioassays to detect activity and isolate piceatannol as the antileukemic principle from the seeds of Euphorbia lagascae. J Natural Prod 1984;47:347-52.

11. Tyagi S, Chirag JP, Raghvendra. Biological and clinical spectrum of piceatannol-a hydroxylated analogue of resveratrol: a phytochemical review. J Biomed Pharm Res 2012;1:1-5.

12. Kukreja A, Wadhwa N, Tiwari A. Therapeutic role of resveratrol and piceatannol in disease prevention. J Blood Disorders Transf 2014;5:1-6.

13. Rossi M, Caruso F, Opazo C, Salciccioli J. Crystal and molecular structure of piceatannol; scavenging features of resveratrol and piceatannol on hydroxyl and peroxyl radicals and docking with transthyretin. J Agric Food Chem 2008;56:10557-66.

14. Ashikawa K, Majumdar S, Banerjee S, Bharti AC, Shishodia S, Aggarwal BB. Piceatannol inhibits TNF-Induced NF- $\kappa B$ activation and NF- $\mathrm{kB}-$ mediated gene expression through suppression of I $\mathrm{kB} \alpha$ Kinase and p65 phosphorylation. J Immunol 2002;169:6490-7.

15. Youn J, Lee JS, Na HK, Kundu JK, Surh YJ. Resveratrol and piceatannol inhibit iNOS expression and NF- $\mathrm{B}$ activation in dextran sulfate sodium-induced mouse colitis. Nutr Cancer 2009;61:847-54.

16. Kalariya NM, Shoebc M, Aramati BM, Reddy ABM, Sawhney R, Ramana KV. Piceatannol suppresses endotoxin-induced ocular inflammation in rats. Int Immpharmacol 2013;17:439-46.

17. Son PS, Park SA, Na HK, Jue DM, Kim S, Surh YJ. Piceatannol, a catechol-type polyphenol, inhibits phorbol ester-induced NF$\mathrm{kB}$ activation and cyclooxygenase-2 expression in human breast epithelial cells: cysteine 179 of IKKb as a potential target. Carcinogenesis 2010;31:1442-9.

18. Farrand L, Byun S, Kim JY, Im-Aram A, Lee J, Lim S, et al. Piceatannol enhances cisplatin sensitivity in ovarian cancer via modulation of 
p53, X-linked Inhibitor of apoptosis protein (XIAP), and mitochondrial fission. J Biol Chem 2013;288:23740-50.

19. Butler AR. The Jaffé reaction. Part II. A kinetic study of the Janovsky complexes formed from creatinine (2-imino-1-methylimazolidin-4one) and acetone. J Chem Soc Perkin Trans 1975;2:853-7.

20. Clark VL, Kruse JA. Clinical methods: the history, physical, and laboratory examinations. JAMA 1990;264:2808-9.

21. Banchroft JD, Stevens A, Turner DR. Theory and practice of histological techniques. 4th ed. Churchil Livingstone, New York; 1996.

22. Park JY, Choi P, Kim T, Ko H, Kim HK, Kang KS, et al. Protective effects of processed ginseng and its active ginsenosides on cisplatin-induced nephrotoxicity: in vitro and in vivo studies. J Agric Food Chem 2015;63:5964-9.

23. Taguchi T, Nazneen A, Abid MR, Razzaque MS. Cisplatin associated nephrotoxicity and pathological events. Contrib Nephrol Basel, Karger 2005;148:107-21.

24. Gautam RK, Singh RK, Ms K. Evaluation of nephroprotective activity of mentha arvensis in cisplatin induced nephrotoxicity. Asian J Pharm Clin Res 2014;7:188-91.

25. Saleh S, Ain-Shoka AA, El-Demerdash E, Khalef MM. Protective effects of the angiotensin II receptor blocker losartan on cisplatininduced kidney injury. Chemotherapy 2009;55:399-406.

26. Arjumand W, Sultana S. Glycyrrhizic acid: a phytochemical with a protective role against cisplatin-induced genotoxicity and nephrotoxicity. Life Sci 2011;89:422-9.

27. Reddy UK, Kumar S. Protective effect of Abutilon indicum L. (Malvaceae) against cisplatin induced nephrotoxicity in rats. Innovare J Life Sci 2013;17:35-9.

28. Yao X, Panichpisal K, Kurtzman N, Nugent K. Cisplatin nephrotoxicity: a review. Am J Med Sci 2007;334:115-24.

29. Huang Q, Dunn RT, Jayadev S, DiSorbo O, Pack FD, Farr SB, et al. Assessment of cisplatin induced nephrotoxocity by microarray technology. Toxicol Sci 2001;63:196-207.
30. Sahu BD, Kumar JM, Sistla R. Baicalein, a bioflavonoid, prevents cisplatin-induced acute kidney injury by upregulating antioxidant defenses and down-regulating the MAPKs and NF- $\kappa$ B pathways. PLoS One 2015;10:1-19.

31. Praharsheta AM, Samuel V, Nirmala P. Role of ebselen, a selenoorgano compound in cisplatin induced nephrotoxicity in wistar rats. Int J Chem Pharm Res 2015;7:57-9.

32. Mondi S, Kvsrg P, Jhansi D, Vijay R. Prophylactic and curative effect of ethanolic extract of bassia malabarica bark against cisplatin induced nephrotoxicity. Asian J Pharm Clin Res 2014;7:143-6.

33. Rhayem $\mathrm{Y}$, Thérond $\mathrm{P}$, Camont L, Couturier $\mathrm{M}$, Beaudeux JL, Legrand A, et al. Chain-breaking activity of resveratrol and piceatannol in a linoleate micellar model. Chem Phys Lipids 2008;155:48-56.

34. Woo A, Min B, Ryoo S. Piceatannol-3'-O- $\beta$-D-glucopyranoside as an active component of rhubarb activates endothelial nitric oxide synthase through inhibition of arginase activity. Exp Mol Med 2010;42:524-32.

35. Abraham E. Nuclear factor- $\kappa \mathrm{B}$ and its role in sepsis-associated organ failure. J Infect Dis 2003;187 Suppl 2:364-9.

36. Oh GS, Kim HJ, Shen AH, Lee SB, Yang SH, Shim H, et al. New therapeutic concept of NAD redox balance for cisplatin nephrotoxicity. Biomed Res Int 2016;1:1-12.

37. Jin CY, Moon DO, Lee KJ, Kim MO, Lee JD, Choi YH. Piceatannol attenuates lipopolysaccharide-induced NF-kappaB activation and NF-kappaB-related proinflammatory mediators in BV2 microglia. Pharmacol Res 2006;54:461-7.

\section{How to cite this article}

- $\quad$ Sara A Wahdan, Samar S Azab, Doaa A Elsherbiny, Ebtehal EL Demerdash. Piceatannol ameliorates cisplatin-induced histological and biochemical alterations in rats kidney. Int J Pharm Pharm Sci 2017;9(10):305-311. 